\title{
Avaliação dos Parâmetros Derivados do Eletroencefalograma durante Administração de Diferentes Concentrações de Óxido Nitroso *
}

\author{
Evaluation of Electroencephalographic Parameters during the \\ Administration of Different Nitrous Oxide Concentrations
}

Sara Lúcia Cavalcante, $T S A^{1}$, Rogean Rodrigues Nunes, $T S A^{2}$

\begin{abstract}
RESUMO
Cavalcante SL, Nunes RR - Avaliação dos Parâmetros Derivados do Eletroencefalograma durante Administração de Diferentes Concentrações de Óxido Nitroso
\end{abstract}

Justificativa e Objetivos - A análise espectral do eletroencefalograma vem sendo usada como medida da profundidade anestésica, nível de hipnose e sedação de diversos agentes anestésicos. O objetivo deste estudo foi avaliar os efeitos do $\mathrm{N}_{2} \mathrm{O}$ sobre os parâmetros derivados do eletroencefalograma, nível de sedação pela Escala Analógica de Alerta e Sedação (EAS), variáveis hemodinâmicas e ventilatórias.

Método - Participaram do estudo 30 pacientes adultos, de ambos os sexos, estado físico ASA I, com idades entre 20 e 40 anos, que se submeteram ao seguinte protocolo: respirar espontaneamente e relaxar de olhos fechados durante dez minutos, sendo coletados dados em três momentos: $\mathrm{M} 1$ - antes da oferta de $\mathrm{N}_{2} \mathrm{O} ; \mathrm{M} 2-\mathrm{N}_{2} \mathrm{O}$ a $30 \%$ em $\mathrm{O}_{2} ; \mathrm{M} 3-\mathrm{N}_{2} \mathrm{O}$ a $50 \%$ em $\mathrm{O}_{2}$. A coleta dos dados de $\mathrm{M} 2$ e M3 foi realizada após quinze minutos da estabilização das frações expiradas de $\mathrm{N}_{2} \mathrm{O}\left(\mathrm{FeN}_{2} \mathrm{O}\right)$ em $30 \%$ e $50 \%$ respectivamente, com o tempo de 5 minutos entre as frações. Os parâmetros estudados foram os seguintes: eletroencefalográficos: BIS, SEF1, SEF2, potência total (PT) e taxa de supressão (TS); hemodinâmicos: freqüência cardíaca $(F C)$, pressão arterial sistólica (PAS), pressão arterial diastólica (PAD); ventilatórios: $\mathrm{SpO}_{2}, \mathrm{P}_{\mathrm{ET}} \mathrm{CO}_{2}$, ventilação minuto e freqüência respiratória; clínicos: os pacientes foram classificados numericamente em ordem decrescente $(5,4,3,2$ e 1) quanto ao seu estado de alerta, pela EAS.

Resultados - O N $\mathrm{N}_{2} \mathrm{O}$ a $30 \%$ e a $50 \%$ modificou estatisticamente o BIS, SEF1, SEF2, PT e o grau de sedação pela EAS, ao longo dos momentos estudados. Não foram observadas modificações clinicamente importantes, pois todos os pacientes mostraram-se cooperativos e levemente sedados, apesar das variações do SEF1 e SEF2 indicarem valores compatíveis com estágio de hipnose profunda. As variações

\footnotetext{
* Recebido do (Received from) Serviço de Anestesiologia do São Lucas Hospital de Cirurgia e Anestesia

1. Coordenadora do Centro de Estudos do São Lucas - Hospital de Cirurgia e Anestesia

2. Diretor Clínico e Chefe do Serviço de Anestesiologia do São Lucas Hospital de Cirurgia e Anestesia
}

Apresentado (Submitted) em 10 de setembro de 2001

Aceito (Accepted) para publicação em 19 de junho de 2002

Correspondência para (Mail to)

Dra. Sara Lúcia Cavalcante

Rua Rocha Lima, 1290/1304

60135-000 Fortaleza, CE

E-mail: saralucia@fortalnet.com.br

(C) Sociedade Brasileira de Anestesiologia, 2003 estatísticas nos parâmetros hemodinâmicos e ventilatórios não foram clinicamente significativas.

Conclusões - $\mathrm{O} \mathrm{N}_{2} \mathrm{O}$ a $30 \%$ e a $50 \%$, em pacientes sem medicação pré-anestésica, induzem a um leve estado de sedação avaliado pela EAS, havendo correspondência com o BIS, o mesmo não ocorrendo com SEF1 e SEF2.

UNITERMOS: ANESTÉSICOS, Gasoso: óxido nitroso; MONITORIZAÇÃO: eletroencefalográfica, índice bispectral, potência total, SEF 95\%, taxa de supressão

\section{SUMMARY}

Cavalcante SL, Nunes RR - Evaluation of Electroencephalographic Parameters during the Administration of Different Nitrous Oxide Concentrations

Background and Objectives - Spectral electroencephalography has been used to measure anesthetic depth, hypnosis and sedation levels induced by different anesthetic agents. This study aimed at evaluating the effects of $\mathrm{N}_{2} \mathrm{O}$ on electroencephalographic (EEG) variables and sedation levels according to Observer Assessment of Alertness/Sedation $(\mathrm{OAA} / \mathrm{S})$ scale, as well as on hemodynamic and respiratory parameters.

Methods - Thirty adult patients from both genders, physical status ASA I, aged 20 and 40 years, were submitted to the following protocol: after 10 minutes of relaxation, spontaneously breathing with eyes close, patients were given 30\% and 50\% $\mathrm{N}_{2} \mathrm{O}$ concentrations under face mask. Data were collected in three moments: M1- before $\mathrm{N}_{2} \mathrm{O}$ administration; $\mathrm{M} 2$ - 30\% $\mathrm{N}_{2} \mathrm{O}$ in $\mathrm{O}_{2} ; \mathrm{M} 3-50 \% \mathrm{~N}_{2} \mathrm{O}$ in $\mathrm{O}_{2}$. Data for $\mathrm{M} 2$ and $\mathrm{M} 3$ were collected 15 minutes after stabilization of $30 \%$ and $50 \% \mathrm{~N}_{2} \mathrm{O}$ expired fractions $\left(\mathrm{FeN}_{2} \mathrm{O}\right)$, respectively, with a 5-minute interval between fractions. The following parameters were evaluated: electroencephalographic: BIS, SEF1, SEF2, power energy $(P E)$ and burst suppression (BS); hemodynamic: heart rate $(H R)$, systolic blood pressure (SBP), diastolic blood pressure $(D B P)$; respiratory: oxygen hemoglobin saturation $\left(\mathrm{SpO}_{2}\right)$, carbon dioxide expired pressure $\left(\mathrm{P}_{\mathrm{ET}} \mathrm{CO}_{2}\right)$, minute ventilation and respiratory rate; clinical: patients were rated in a descending or$\operatorname{der}(5,4,3,2$ and 1) according to OAA/S scale.

Results $-\mathrm{N}_{2} \mathrm{O}$ administrated at $30 \%$ and $50 \%$ concentrations has statistically changed BIS, SEF1, SEF2, PE and OAA/S level of sedation in the studied moments. No clinically important changes were observed, as all patients were cooperative and slightly sedated, though SEF1 and SEF2 indications of deep hypnosis. Hemodynamic and respiratory parameters changes were not statistically significant.

Conclusions - $\mathrm{N}_{2} \mathrm{O}$ at $30 \%$ and $50 \%$ concentrations in non-premedicated patients has induced mild sedation according to OAA/S scale. There has been correspondence with BIS, but not with SEF1 and SEF2.

KEY WORDS: ANESTHETICS, Gaseous, nitrous oxide; MONITORING: electroencephalography: bispectral index, total power, SEF 95\%, burst suppression 


\section{INTRODUÇÃO}

O óxido nitroso $\left(\mathrm{N}_{2} \mathrm{O}\right)$ é usado em anestesia geral balanceada e em sedação consciente para procedimentos médicos e odontológicos ${ }^{1-3}$. Seus efeitos anestésicos, analgésicos em concentrações sub-anestésicas, amnésicos e psíquicos têm permitido um melhor conhecimento clínico das indicações, contra-indicações e precauções no uso deste gás ${ }^{4-7}$. Outras características do $\mathrm{N}_{2} \mathrm{O}$, como a capacidade de ativar o sistema nervoso simpático ${ }^{8,9}$, aumentar o tônus muscular ${ }^{10}$ e acelerar a indução dos anestésicos inalatórios ${ }^{11}$ também estão descritas. A análise bispectral do eletroencefalograma (BIS) vem sendo usada como método para averiguar os efeitos de agentes inalatórios sobre a profundidade anestésica ${ }^{12}$ e o nível de sedação e de hipnose determinado por drogas venosas, como o propofol ${ }^{13}$, midazolam ${ }^{14}$, opióides ${ }^{14}$ e agonistas $\alpha_{2}$-adrenérgicos ${ }^{15}$. $\mathrm{O}$ presente estudo teve por objetivo avaliar os efeitos do $\mathrm{N}_{2} \mathrm{O}$ sobre os parâmetros derivados do eletroencefalograma, nível de sedação através da Escala Analógica de Alerta e Sedação $(E A S)^{16}$, variáveis hemodinâmicas e ventilatórias .

\section{MÉTODO}

Após aprovação do Comitê de Ética e o consentimento formal, participaram do estudo 30 pacientes de ambos os sexos, com idades entre 20 e 40 anos, estado físico ASA l e índice de massa corpórea entre 22 e 28 . Foram excluídos pacientes em uso de drogas psicoativas ou passado de doenças psiquiátricas. Nenhum dos pacientes fez uso de medicação pré-anestésica. Na sala de operação, após venóclise, procederam-se as monitorizações da pressão arterial pelo método não invasivo, oximetria de pulso e cardioscópio em $D_{\|}$e V5. A análise dos parâmetros derivados do eletroencefalograma foi feita após montagem de eletrodos de cloreto de prata, utilizando-se dois canais (F7, F8), um terra (Fp1) e um de referência (Fz). Uma máscara facial com dispositivo adequado permitiu perfeito acoplamento máscara-face, utilizando um sistema circular com absorvedor de $\mathrm{CO}_{2}$, para ventilação pulmonar. As concentrações inspiradas e expiradas de oxigênio $\left(\mathrm{O}_{2}\right)$ e $\mathrm{N}_{2} \mathrm{O}$, e da pressão expirada de dióxido de carbono $\left(\mathrm{P}_{\mathrm{ET}} \mathrm{CO}_{2}\right)$ foram monitorizadas continuamente, assim como a freqüência respiratória, através do analisador de agentes anestésicos. A ventilação por minuto foi registrada por um espirômetro no ramo expiratório do circuito respiratório. Os pacientes foram orientados a fechar os olhos e relaxar, concentrando-se na respiração durante dez minutos, para a seguir coletarem-se os dados em três momentos: $\mathrm{M} 1$ antes da oferta de $\mathrm{N}_{2} \mathrm{O} ; \mathrm{M} 2-\mathrm{N}_{2} \mathrm{O}$ a $30 \%$ em $\mathrm{O}_{2} ; \mathrm{M} 3-\mathrm{N}_{2} \mathrm{O}$ a $50 \%$ em $\mathrm{O}_{2}$. Acoleta dos dados de $\mathrm{M} 2$ e M3 foi realizada após quinze minutos da estabilização das frações expiradas de $\mathrm{N}_{2} \mathrm{O}\left(\mathrm{FeN}_{2} \mathrm{O}\right)$ em $30 \%$ e a $50 \%$ respectivamente. O tempo de ajuste de $30 \%$ e $50 \%$ da $\mathrm{FeN}_{2} \mathrm{O}$ foi estabelecido em cinco minutos.

Os parâmetros estudados foram os seguintes: a) eletroencefalográficos: BIS, SEF1, SEF2, potência total (PT), definida como a medida total absoluta de potência na faixa de fre- qüência de 0,5 a $30 \mathrm{~Hz}$, sendo seus valores de variação compreendidos entre 40 e $100 \mathrm{db}^{17}$; b) taxa de supressão (TS), definida como percentual de tempo nos últimos 63 segundos em que o EEG registrou amplitudes inferiores a $5 \mu \mathrm{V}$ (baixa amplitude compatível com anestesia profunda) ${ }^{18}$; c) hemodinâmicos: freqüência cardíaca (FC), pressão arterial sistólica (PAS), pressão arterial diastólica (PAD). Os parâmetros hemodinâmicos considerados limites inferiores no protocolo foram PAS $=80 \mathrm{mmHg}$ e PAD $=60 \mathrm{mmHg}$. Os limites superiores de PAS e PAD foram considerados clinicamente importantes acima de $20 \%$ dos valores em M1. AFC com variações maiores que $20 \%$ em relação aos valores em M1 foram consideradas de significância clínica; d) ventilatórios: saturação periférica de hemoglobina $\left(\mathrm{SpO}_{2}\right)$, pressão expirada de dióxido de carbono $\left(\mathrm{P}_{\mathrm{ET}} \mathrm{CO}_{2}\right)$, ventilação minuto $(\mathrm{VM})$, freqüência respiratória (FR); e) clínicos: os pacientes foram classificados numericamente em ordem decrescente $(5,4,3,2$ e 1$)$ quanto ao seu estado de alerta, seguindo a Escala Analógica de Sedação (EAS) ${ }^{16}$ onde:

5 - Resposta imediata quando solicitado pelo nome. Fala normal, expressão facial normal e olhos abertos sem queda das pálpebras;

4 - Resposta letárgica quando solicitado pelo nome; fala lenta, expressão facial relaxada e olhos embaçados ou com discreta ptose palpebral;

3 - Resposta somente quando solicitado pelo nome em voz alta ou repetidas vezes. Fala com pronúncia indistinta, ininteligível, expressão facial relaxada (queda da mandíbula) e olhos embaçados com ptose;

2 - Resposta somente a estímulos táteis; fala com poucas palavras compreensíveis;

1 - Não responde a estímulos táteis.

Os parâmetros estudados foram representados por média e desvio padrão e submetidos a tratamento estatístico através da análise de variância e posterior aplicação do teste de Tukey. Adotou-se o nível de significância de 0,05 (5\%). Níveis descritivos $(p)$ inferiores a este valor foram considerados significantes.

\section{RESULTADOS}

Os dados demográficos da amostra estudada estão apresentados na tabela I e foram considerados homogêneos. As misturas $\mathrm{O}_{2}+\mathrm{N}_{2} \mathrm{O}$ a $30 \%$ e $\mathrm{O}_{2}+\mathrm{N}_{2} \mathrm{O}$ a $50 \%$ foram bem toleradas por todos os pacientes. Discreta agitação transitória foi observada em três pacientes que inalaram $\mathrm{O}_{2}+\mathrm{N}_{2} \mathrm{O}$ a $50 \%$. A análise das médias do BIS, SEF1 e SEF2 demonstra diferença significativa ao longo do tempo: $M 1>M 2>M 3(p<0,05$ entre todos os momentos) (Figura 1). ATS foi significativamente diferente com M1 $<M 2<M 3(p<0,05$ entre todos os momentos) (Figura 2). A TS permaneceu em zero ao longo dos momentos estudados. A variação no grau de sedação pela EAS foi significativa e mostrou M1 $>M 2>M 3(p<0,05$ entre todos os momentos) (Tabela II). Os valores da PAS, PAD e FC nos momentos estudados são significativamente difeVol. 53, Nº 1, Janeiro - Fevereiro, 2003 
rentes ao longo do estudo com M3 $>M 2>M 1(p<0,05)$; entretanto, não ultrapassaram os limites pré-estabelecidos no protocolo (Tabela II e Figura 3). Na tabela III estão descritas as médias e desvios padrões referentes a $\mathrm{SpO}_{2}, \mathrm{P}_{\mathrm{ET}} \mathrm{CO}_{2}, \mathrm{VM}$ e FR. A $\mathrm{P}_{\mathrm{ET}} \mathrm{CO}_{2}$ variou de modo estatisticamente significativo em que $M 3<M 2<M 1(p<0,05)$, sem representação clínica significativa (Figura 4). Avariação na FR também foi significativa ao longo do protocolo com M3 $>M 2>M 1(p<0,05$ entre todos os momentos), porém clinicamente inexpressiva (Figura 5). OVM demonstrou M1 = M2 $<M 3(p<0,05)$ (Figura 5). A $\mathrm{SpO}_{2}$ apresentou comportamento estatístico semeIhante entre $\mathrm{M} 1$ e $\mathrm{M} 2$ e entre M2 e M3. Porém, a SpO 2 de M3 foi significativamente maior que $M 1(p<0,05)$ (Figura 4).

Tabela I - Variáveis Idade, Peso e Altura (Média \pm DP)

\begin{tabular}{lccc}
\hline Sexo & Idade (anos) & Peso $(\mathrm{kg})$ & Altura $(\mathrm{cm})$ \\
\hline Feminino & $32,18 \pm 4,35$ & $61,70 \pm 4,14$ & $157,70 \pm 4,05$ \\
Masculino & $25,00 \pm 1,73$ & $64,66 \pm 9,38$ & $163,66 \pm 9,81$ \\
\hline
\end{tabular}

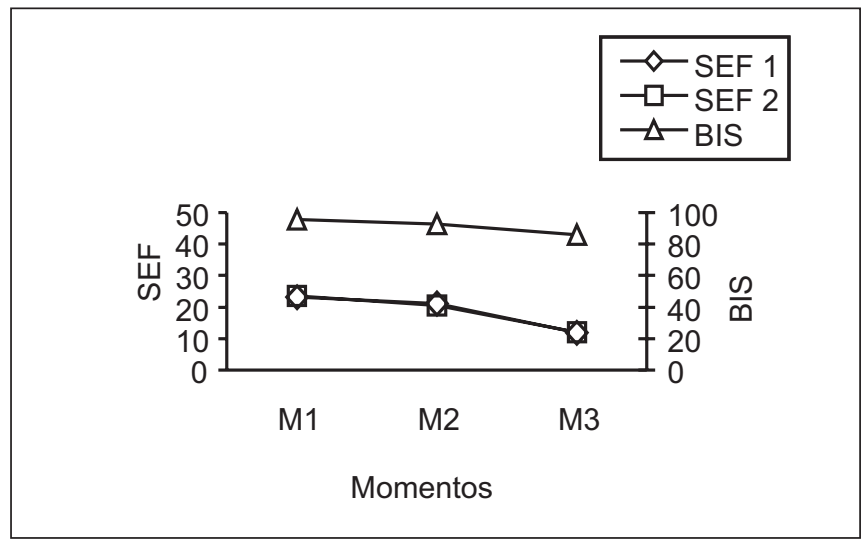

Figura 1 - Médias de SEF e BIS em Pacientes Tratados com $\mathrm{N}_{2} \mathrm{O}$ $M 1>M 2>M 3(p<0,05$ entre todos os momentos)

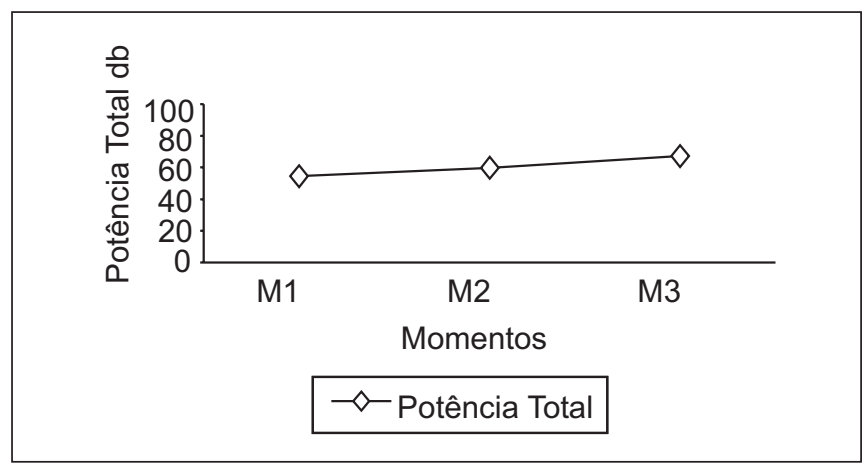

Figura 2 - Médias de Potência Total (PT) em Pacientes Tratados $\operatorname{com~} \mathrm{N}_{2} \mathrm{O}$ $\mathrm{M} 1<\mathrm{M} 2<\mathrm{M} 3$ ( $p<0,05$ entre todos os momentos)
Tabela II - Pressão Arterial Sistólica (PAS), Pressão Arterial Diastólica (PAD), Freqüência Cardíaca (FC) e Escala Analógica de Alerta e Sedação (EAS). (Média $\pm D P$ )

\begin{tabular}{lcccc}
\hline Momentos & PAS & PAD & FC & EAS \\
\hline M1 & $117,50 \pm 6,14$ & $73,23 \pm 4,57$ & $71,80 \pm 7,16$ & $5,00 \pm 0,00$ \\
M2 & $126,43 \pm 4,59$ & $78,23 \pm 4,62$ & $74,43 \pm 5,39$ & $4,76 \pm 0,43$ \\
M3 & $136,46 \pm 5,02$ & $82,93 \pm 3,02$ & $81,36 \pm 6,51$ & $3,96 \pm 0,18$ \\
\hline
\end{tabular}

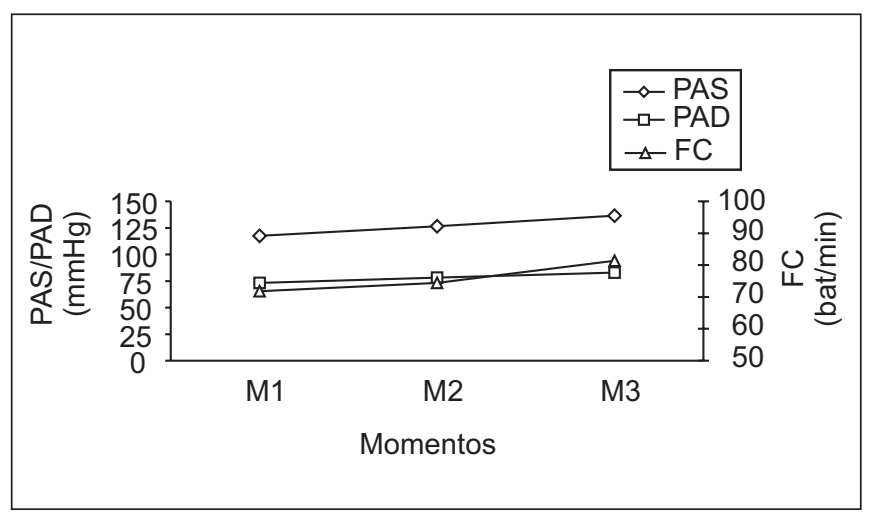

Figura 3 - Médias de Pressão Sistólica (PAS) e Diastólica (PAD) e Freqüência Cardíaca ( $F C$ ) em Pacientes Tratados com $\mathrm{N}_{2} \mathrm{O}$ M3 > M2 > M1 $(p<0,05)$

Tabela III - Saturação Periférica de Hemoglobina $\left(\mathrm{SpO}_{2}\right)$, Pressão Expirada de Dióxido de Carbono $\left(\mathrm{P}_{\mathrm{ET}} \mathrm{CO}_{2}\right)$, Volume Minuto (VM), Freqüência Respiratória (FR). (Média $\pm \mathrm{DP}$ )

\begin{tabular}{lcccc}
\hline Momentos & $\mathrm{SpO}_{2}$ & $\mathrm{P}_{\mathrm{ET}} \mathrm{CO}_{2}$ & $\mathrm{VM}$ & $\mathrm{FR}$ \\
\hline M1 & $99,26 \pm 0,86$ & $38,63 \pm 2,14$ & $5,64 \pm 1,67$ & $10,56 \pm 1,27$ \\
M2 & $99,56 \pm 0,50$ & $35,63 \pm 1,42$ & $5,61 \pm 1,79$ & $13,70 \pm 3,57$ \\
M3 & $95,70 \pm 0,62$ & $33,33 \pm 1,24$ & $9,82 \pm 1,55$ & $18,5 \pm 2,43$ \\
\hline
\end{tabular}

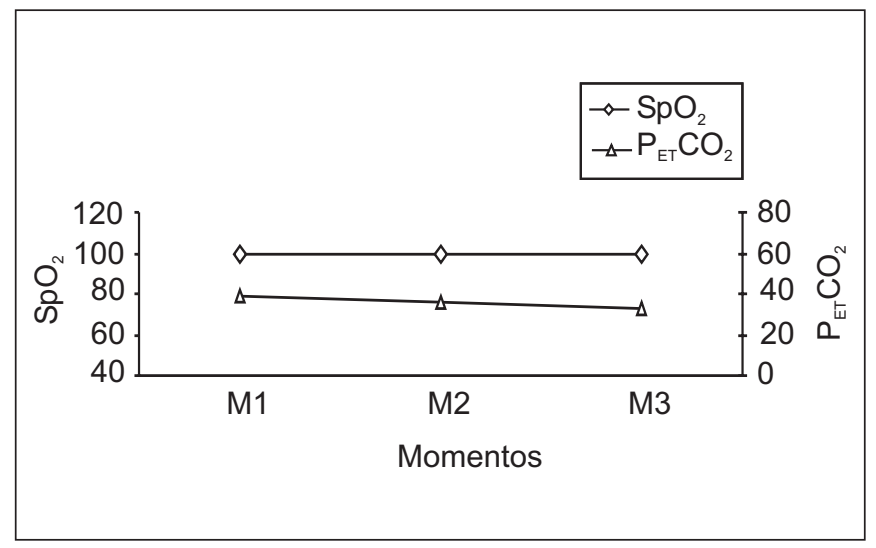

Figura 4 - Médias de $\mathrm{SpO}_{2}$ e $\mathrm{P}_{\mathrm{ET}} \mathrm{CO}_{2}$ em Pacientes Tratados com $\mathrm{N}_{2} \mathrm{O}, \mathrm{SpO}_{2} \mathrm{M} 3>\mathrm{M} 1(\mathrm{p}<0,05) . \mathrm{P}_{\mathrm{ET}} \mathrm{CO}_{2} \mathrm{M} 3<\mathrm{M} 2<\mathrm{M} 1(\mathrm{p}<0,05)$ 


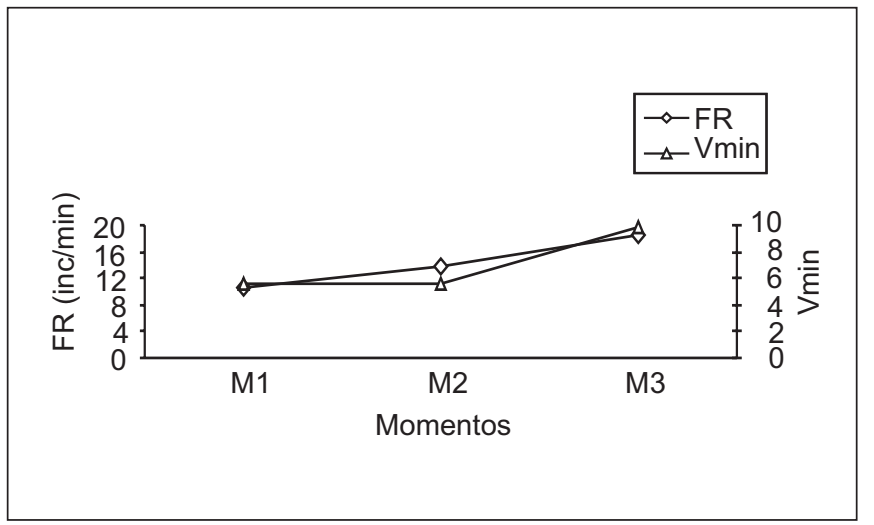

Figura 5 - Médias de Freqüência Respiratória (FR) e Volume Minuto (VM) em Pacientes Tratados com $\mathrm{N}_{2} \mathrm{O}$. FR: M3 > M2 > M1 $(p<0,05)$ entre todos os momentos. VM: M1 = M2 $<M 3$ $(\mathrm{p}<0,05)$

\section{DISCUSSÃO}

O $\mathrm{N}_{2} \mathrm{O}$ é um gás inodoro, não inflamável, com propriedades físico-químicas próprias de um anestésico pouco potente, usado em altas concentrações ${ }^{4}$. Devido a estas características, o fluxo de gás que passa dos alvéolos para a circulação pulmonar durante a indução é mais rápido e superior ao fluxo de gás no sentido inverso ${ }^{4}$. A fração alveolar alcança $90 \%$ da fração inspirada em menos de cinco minutos e a fração cerebral em menos de sete minutos ${ }^{4}$. Estes dados explicam os tempos empregados neste trabalho: cinco minutos de ajuste na concentração do $\mathrm{N}_{2} \mathrm{O}$ e somente quando completados quinze minutos da fração expirada do $\mathrm{N}_{2} \mathrm{O}\left(\mathrm{FeN}_{2} \mathrm{O}\right)$ é que se procedeu a tomada dos dados.

Estudo mostra quatro efeitos centrais do $\mathrm{N}_{2} \mathrm{O}$ : psíquico, amnésico, analgésico e cognitivo em diferentes concentrações: $5 \%$ a $25 \%, 26 \%$ a $45 \%, 46 \%$ a $65 \%$ e $68 \%$ a $85 \%$, respectivamente ${ }^{19}$.

Na prática clínica, encontramos dificuldade em utilizar concentrações acima de $50 \%$, pela alta incidência de efeitos colaterais como náuseas, ansiedade e vômitos, também documentados por outros autores ${ }^{7}$. Outro estudo mostrou uma pequena redução no grau de responsividade, classificada por desorientação, com $\mathrm{N}_{2} \mathrm{O}$ a $30 \%{ }^{20}$. Outros autores observaram que o $\mathrm{N}_{2} \mathrm{O}$ a $20 \%$ e a $30 \%$ causaram prejuízo nos níveis de memória explícita, consciência imediata e sensações subjetivas ${ }^{21,22}$.

As variações encontradas na EAS com ambas concentrações ( $30 \%$ e $50 \%$ ) não foram clinicamente importantes, uma vez que todos os pacientes mantiveram-se conscientes, capazes de responder a comandos verbais.
Estas observações estão de acordo com os trabalhos de outros autores ${ }^{23}$, os quais mostraram que a CAM - acordada do $\mathrm{N}_{2} \mathrm{O}$ é 0,64 CAM e que nesta concentração a consciência não é completamente abolida. As variações no BIS são compatíveis com o fraco efeito sedativo do $\mathrm{N}_{2} \mathrm{O}$ a $30 \%$ e $50 \%$. Aanálise de SEF1 e SEF2 demonstrou maior densidade de potência em bandas de freqüência baixa, mostrando que o $\mathrm{N}_{2} \mathrm{O}$ apresentou importante efeito lentificador da freqüência das ondas cerebrais. A potência total, outro parâmetro eletroencefalográfico, teve comportamento estatístico semelhante ao descrito por outros autores, que demonstraram um aumento da mesma, à medida que a anestesia se aprofunda ${ }^{17}$. Valores diferentes de zero na derivada da taxa de supressão não foram observados em nenhum dos pacientes, mostrando que nestas concentrações, o $\mathrm{N}_{2} \mathrm{O}$ não é capaz de reduzir a amplitude da voltagem do EEG, abaixo de $5 \mu \mathrm{V}^{18}$.

As variações de PAS, PAD, $\mathrm{FC}$ e $\mathrm{SpO}_{2}$ nos momentos estudados não ultrapassaram os limites normais pré-estabelecidos no protocolo.

Os efeitos do $\mathrm{N}_{2} \mathrm{O}$ sobre os parâmetros respiratórios coincidiram com a descrição clássica deste anestésico sobre a ventilação espontânea com aumento da freqüência respiratória com diminuição da $\mathrm{P}_{\mathrm{ET}} \mathrm{CO}_{2}{ }^{4}$. O presente trabalho diferiu quanto ao volume minuto, que aumentou significativamente ao longo dos momentos.

As misturas de $\mathrm{N}_{2} \mathrm{O}$ a $30 \%$ em $\mathrm{O}_{2}$ e $\mathrm{N}_{2} \mathrm{O}$ a $50 \%$ em $\mathrm{O}_{2}$, em pacientes sem medicação pré-anestésica, determinaram variações estatisticamente significativas no BIS, SEF1, SEF2, potência total e EAS. As variações mais significativas ocorreram no SEF1 e SEF2, tendo atingido valores correspondentes a planos profundos de hipnose, não representando, no entanto, uma correspondência com a avaliação clínica feita com base na EAS, nem com a variação do BIS. Hans e col. ${ }^{24}$, em recente estudo, demonstraram que durante procedimento cirúrgico, o óxido nitroso $(20 \%, 40 \%$ e $60 \%)$ produz redução significativa dose-dependente no BIS e SEF 95\%, sem importantes alterações nos parâmetros hemodinâmicos (PA e FC). Entretanto, os autores associaram ao $\mathrm{N}_{2} \mathrm{O}$, opióide peridural, sevoflurano, após indução com propofol e sufentanil, o que dificulta uma melhor validação dos resultados obtidos.

Concluindo, nas condições empregadas, o $\mathrm{N}_{2} \mathrm{O}$ a $30 \%$ e a $50 \%$ resultaram em fraco efeito sedativo, não abolindo a consciência. As variações no BIS foram compatíveis com as mudanças na EAS, o mesmo não ocorrendo com SEF1 e SEF2, que não se mostraram válidos como índices quantitativos do grau de hipnose na técnica empregada, podendo no entanto, refletir um possível componente analgésico do óxido nitroso. 


\section{Evaluation of Electroencephalographic Parameters during the Administration of Different Nitrous Oxide Concentrations}

\author{
Sara Lúcia Cavalcante TSA, M.D., Rogean Rodrigues Nunes \\ TSA, M.D.
}

\section{INTRODUCTION}

Nitrous oxide $\left(\mathrm{N}_{2} \mathrm{O}\right)$ is commonly employed in balanced general anesthesia and conscious sedation for medical and dental procedures ${ }^{1-3}$. Its anesthetic, analgesic, amnesic and psychic effects in sub-anesthetic concentrations have allowed for a better clinical knowledge of indications, counterindications and precautions with the use of such gas ${ }^{4-7}$. Other $\mathrm{N}_{2} \mathrm{O}$ features, such as the ability to activate the sympathetic nervous system ${ }^{8,9}$, to increase muscle tone ${ }^{10}$ and to accelerate inhalational anesthetics induction ${ }^{11}$, have also been described. Bispectral electroencephalography (BIS) has been used to investigate the effects of inhalational anesthetics on anesthetic depth ${ }^{12}$ and the level of sedation and hypnosis induced by intravenous drugs such as propofol ${ }^{13}$, midazolam ${ }^{14}$, opioids ${ }^{14}$ and $\alpha_{2}$-adrenergic agonists ${ }^{16}$.

This study aimed at evaluating $\mathrm{N}_{2} \mathrm{O}$ effects on electroencephalographic parameters and sedation levels according to Observer Assessment of Alertness/Sedation (OAA/S) scale ${ }^{16}$, as well as hemodynamic and ventilatory variables.

\section{METHODS}

After the Ethics Committee approval and their formal consent, 30 patients of both genders, aged 20 to 40 years, physical status ASA I and body mass index between 22 and 28 were included in the study. Patients under psychoactive drugs or with history of psychiatric disease were excluded from the study. Patients were not premedicated. In the operating room and after venoclysis, monitoring was installed consisting of non-invasive blood pressure, pulse oximetry and cardioscopy at $D_{\|}$and V5. EEG-derived parameters were analyzed after the placement of silver chloride leads using two channels (F7, F8), one ground (Fp1) and one reference $(F z)$. An adequate mask was perfectly coupled to patients face, and circle system with $\mathrm{CO}_{2}$ absorber allowed for pulmonary ventilation. Oxygen and $\mathrm{N}_{2} \mathrm{O}$ inspired and expired fractions and carbon dioxide expired pressure $\left(\mathrm{P}_{\mathrm{ET}} \mathrm{CO}_{2}\right)$ were continuously monitored through an anesthetic agents analyzer, as well as the respiratory rate through an anesthetic. Minute ventilation was recorded by a spirometer at the expiratory branch of the respiratory circuit. Patients were asked to close their eyes and relax, concentrating on their breathing for 10 minutes. Then, data were collected in three moments: $\mathrm{M} 1$ - before $\mathrm{N}_{2} \mathrm{O}$ administration; $\mathrm{M} 2-30 \% \mathrm{~N}_{2} \mathrm{O}$ in $\mathrm{O}_{2}$; $\mathrm{M} 3-50 \% \mathrm{~N}_{2} \mathrm{O}$ in $\mathrm{O}_{2}$. Data for $\mathrm{M} 2$ and $\mathrm{M} 3$ were collected 15 minutes after $30 \%$ and $50 \% \mathrm{~N}_{2} \mathrm{O}$ expired fractions $\left(\mathrm{FeN}_{2} \mathrm{O}\right)$ stabilization. Adjustment time for $30 \%$ and $50 \% \mathrm{FeN}_{2} \mathrm{O}$ was established in five minutes.

The following parameters were evaluated: a) electroencephalographic: BIS SEF1, SEF2 and power energy (PE), defined as total absolute potency measurement in the frequency range of 0.5 to $30 \mathrm{~Hz}$, with variation values between 40 and $100 \mathrm{db}^{17}$; b) burst suppression (BS), defined as time percentage in the last 63 seconds in which EEG recorded amplitudes below $5 \mu \mathrm{V}$ (low amplitude compatible with deep anesthesia) ${ }^{18}$; c) hemodynamic: heart rate $(H R)$, systolic blood pressure (SBP) and diastolic blood pressure (DBP). Lowest hemodynamic parameters levels were SBP = $80 \mathrm{mmHg}$ and DBP $=60 \mathrm{mmHg}$. High SBP and DBP values were considered clinically important when above $20 \%$ of $M 1$ values. HR with values above $20 \%$ of $M 1$ was considered clinically important; d) ventilatory: oxygen hemoglobin saturation $\left(\mathrm{SpO}_{2}\right)$, carbon dioxide expired pressure $\left(\mathrm{P}_{\mathrm{ET}} \mathrm{CO}_{2}\right)$, minute ventilation (MV) and respiratory rate (RR); e) clinical: patients were rated in a descending order $(5,4,3,2$ and 1) according to OAA/S scale ${ }^{16}$, where:

5 - Immediate response when called by name. Normal speech, normal facial expression and eyes open without palpebral ptosis;

4 - Lethargic response when called by name; slow speech, relaxed facial expression and dim eyes or mild palpebral ptosis;

3 - Response only when called loudly or repeatedly by name or. Indistinct unintelligible speech, relaxed facial expression (mandible drop), dim eyes and ptosis;

2 - Response only to tactile stimulations; speech with few understandable words;

1 - No response to tactile stimulations.

Parameters were presented in means and standard deviations and submitted to statistical analysis using analysis of variance and Tukey's test. Significance level was established to $0.05(5 \%)$. Descriptive levels $(p)$ below this value were considered significant.

\section{RESULTS}

Demographics data are shown in table I and were considered homogeneous. $30 \% \mathrm{~N}_{2} \mathrm{O}+\mathrm{O}_{2}$ and $50 \% \mathrm{~N}_{2} \mathrm{O}+\mathrm{O}_{2}$ mixtures were well tolerated by all patients. Mild transient agitation was observed in three patients receiving $50 \% \mathrm{~N}_{2} \mathrm{O}+\mathrm{O}_{2}$. BIS, SEF1 and SEF2 analysis showed statistically significant differences along time: $M 1>M 2>M 3(p<0.05$ among all moments) (Figure 1). BS was significantly different, with $\mathrm{M} 1<$ $M 2<M 3$ ( $p<0.05$ among all moments) (Figure 2). BS remained zero throughout all moments. Sedation level variations according to $\mathrm{OAA} / \mathrm{S}$ were significant, with $\mathrm{M} 1>\mathrm{M} 2>\mathrm{M} 3$ ( $p<0.05$ among all moments) (Table II). SBP, DBP and HR were significantly differentalong the study, with $\mathrm{M} 3>\mathrm{M} 2>\mathrm{M} 1$ $(p<0.05)$, however not going beyond limits pre-established by the protocol (Table II and Figure 3). Table III shows $\mathrm{SpO}_{2}$, 
$\mathrm{P}_{\mathrm{ET}} \mathrm{CO}_{2}, \mathrm{MV}$ and RR means and standard deviations. $\mathrm{P}_{\mathrm{ET}} \mathrm{CO}_{2}$ varied significantly, with $M 3<M 2<M 1(p<0.05)$, but no clinical relevance (Figure 4). RR variation was also significant throughout the protocol, with M3 $>M 2>M 1(p<0.05$ among all moments), but also clinically unexpressive (Figure 5). MV has shown $\mathrm{M} 1=\mathrm{M} 2<\mathrm{M} 3(\mathrm{p}<0.05)$ (Figure 5$). \mathrm{SpO}_{2}$ was statistically similar between $\mathrm{M} 1$ and $\mathrm{M} 2$ and between $\mathrm{M} 2$ and $\mathrm{M} 3$. However, $\mathrm{M}_{3} \mathrm{SpO}_{2}$ was significantly higher than $\mathrm{M} 1(\mathrm{p}<$ 0.05) (Figure 4).

Table I - Demographics Data (Mean \pm SD)

\begin{tabular}{lccc}
\hline Gender & Age (years) & Weight $(\mathrm{kg})$ & Height $(\mathrm{cm})$ \\
\hline Female & $32.18 \pm 4.35$ & $61.70 \pm 4.14$ & $157.70 \pm 4.05$ \\
Male & $25.00 \pm 1.73$ & $64.66 \pm 9.38$ & $163.66 \pm 9.81$ \\
\hline
\end{tabular}

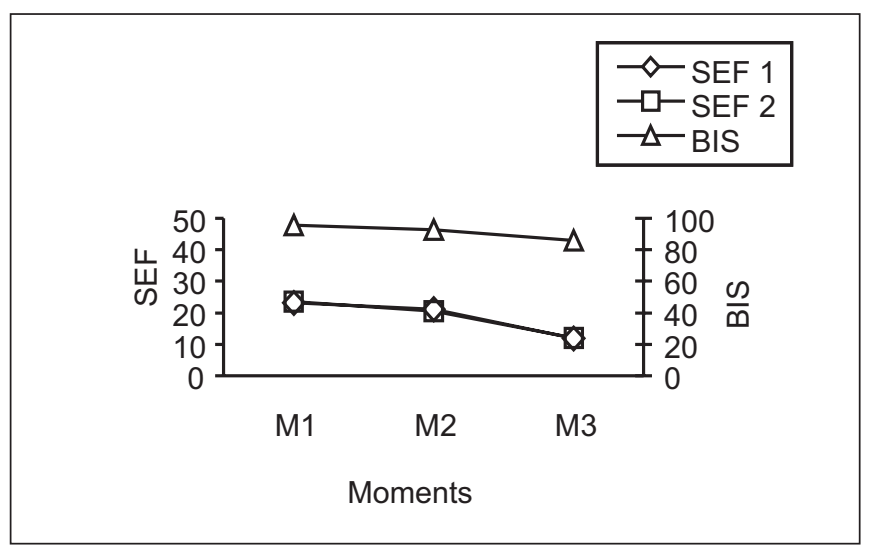

Figure 1 - SEF and BIS Means in Patients Treated with $\mathrm{N}_{2} \mathrm{O}$ $\mathrm{M} 1>\mathrm{M} 2>\mathrm{M} 3$ ( $p<0.05$ among all moments $)$

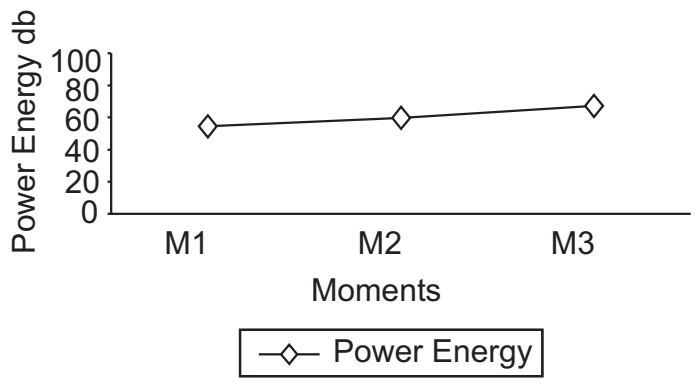

Figure 2 - Power Energy (PE) Means in Patients Treated with $\mathrm{N}_{2} \mathrm{O}$ $\mathrm{M} 1<\mathrm{M} 2<\mathrm{M} 3$ ( $\mathrm{p}<0.05$ among all moments)
Table II - Systolic Blood Pressure (SBP), Diastolic Blood Pressure (DBP) Heart Rate (HR) and Observer Assessment of Alertness/Sedation (OAA/S) Scale. (Mean $\pm \mathrm{SD}$ )

\begin{tabular}{lcccc}
\hline Moments & SBP & DBP & HR & OAA/S \\
\hline M1 & $117.50 \pm 6.14$ & $73.23 \pm 4.57$ & $71.80 \pm 7.16$ & $5.00 \pm 0.00$ \\
M2 & $126.43 \pm 4.59$ & $78.23 \pm 4.62$ & $74.43 \pm 5.39$ & $4.76 \pm 0.43$ \\
M3 & $136.46 \pm 5.02$ & $82.93 \pm 3.02$ & $81.36 \pm 6.51$ & $3.96 \pm 0.18$ \\
\hline
\end{tabular}

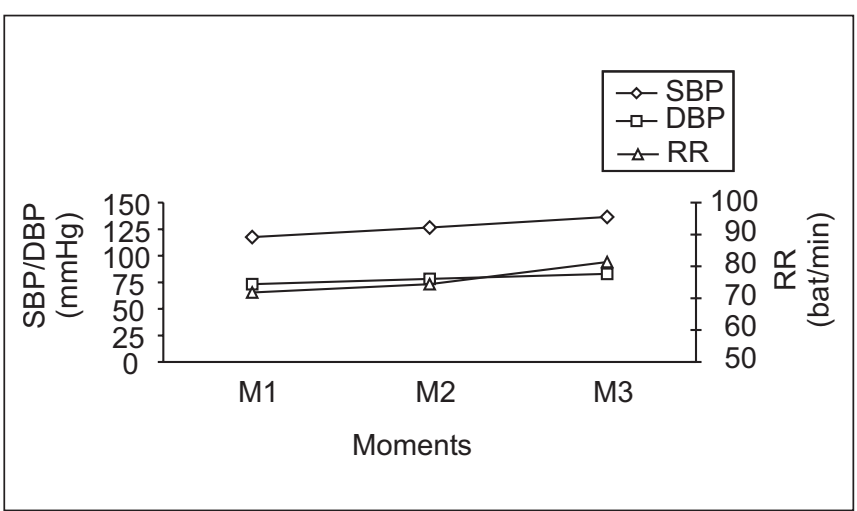

Figure 3 - Systolic (SBP) and Diastolic (DBP) Blood Pressure Means in Patients Treated with $\mathrm{N}_{2} \mathrm{O}$ M3 > M $2>$ M1 $(p<0.05)$

Table III - Oxygen Hemoglobin Saturation $\left(\mathrm{SpO}_{2}\right)$, Carbon Dioxide Expired Pressure $\left(\mathrm{P}_{\mathrm{ET}} \mathrm{CO}_{2}\right)$, Minute Volume (MV), Respiratory Rate $(\mathrm{RR})$. (Mean $\pm \mathrm{SD}$ )

\begin{tabular}{lcccc}
\hline Moments & $\mathrm{SpO}_{2}$ & $\mathrm{P}_{\mathrm{ET}} \mathrm{CO}_{2}$ & $\mathrm{MV}$ & $\mathrm{RR}$ \\
\hline $\mathrm{M} 1$ & $99.26 \pm 0.86$ & $38.63 \pm 2.14$ & $5.64 \pm 1.67$ & $10.56 \pm 1.27$ \\
$\mathrm{M} 2$ & $99.56 \pm 0.50$ & $35.63 \pm 1.42$ & $5.61 \pm 1.79$ & $13.70 \pm 3.57$ \\
$\mathrm{M} 3$ & $95.70 \pm 0.62$ & $33.33 \pm 1.24$ & $9.82 \pm 1.55$ & $18.5 \pm 2.43$ \\
\hline
\end{tabular}

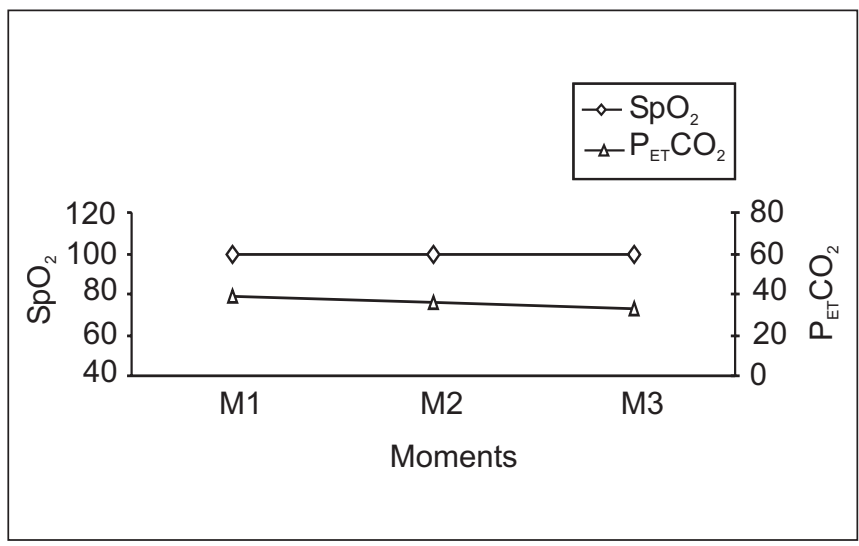

Figure $4-\mathrm{SpO}_{2}$ and $\mathrm{P}_{\mathrm{ET}} \mathrm{CO}_{2}$ Means in Patients Treated with $\mathrm{N}_{2} \mathrm{O}$ $\mathrm{SpO}_{2} \mathrm{M} 3>\mathrm{M} 1(\mathrm{p}<0.05) ; \mathrm{P}_{\mathrm{ET}} \mathrm{CO}_{2} \mathrm{M} 3<\mathrm{M} 2<\mathrm{M} 1(\mathrm{p}<0.05)$

Revista Brasileira de Anestesiologia Vol. 53, № 1, Janeiro - Fevereiro, 2003 


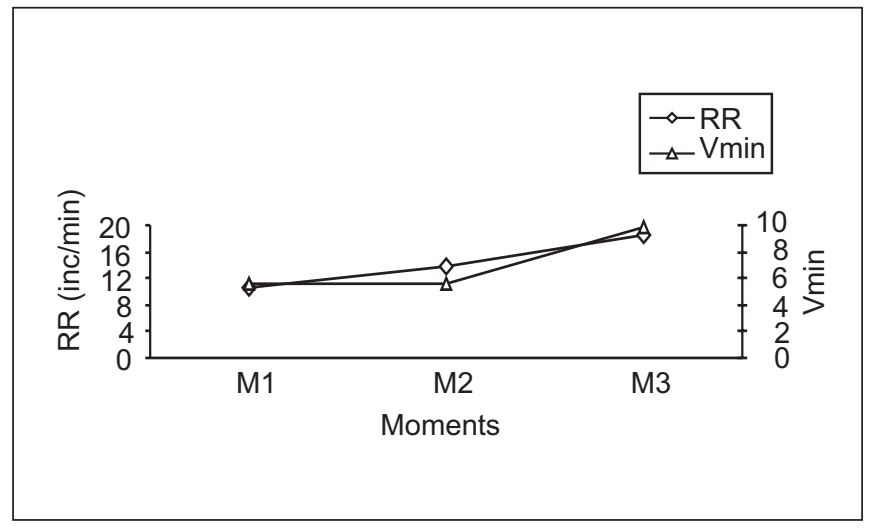

Figure 5 - Respiratory Rate (RR) and Minute Volume (MV) Means in Patients Treated with $\mathrm{N}_{2} \mathrm{O}$; RR: $\mathrm{M} 3>\mathrm{M} 2>\mathrm{M} 1$ $(p<0.05)$ among all moments. MV: M1 = M2 < M3 $(p<0.05)$

\section{DISCUSSION}

$\mathrm{N}_{2} \mathrm{O}$ is an inodorous non-inflammable gas, with physical-chemical characteristics of a low potency anesthetic agent, thus used in high concentrations ${ }^{4}$. Due to such characteristics, gas flow from the alveoli to pulmonary circulation during induction is higher than gas flow in the opposite direction ${ }^{4}$. Alveolar fraction reaches $90 \%$ of the inspired fraction in less than 5 minutes, while brain fraction reaches this value in less than 7 minutes ${ }^{4}$. These data explain the intervals used in this study: 5 minutes of $\mathrm{N}_{2} \mathrm{O}$ concentration adjustment and data collection only 15 minutes after $\mathrm{N}_{2} \mathrm{O}$ expired fraction $\left(\mathrm{FeN}_{2} \mathrm{O}\right)$.

A study has shown four central $\mathrm{N}_{2} \mathrm{O}$ effects: psychic, amnesic, analgesic, and cognitive, in different concentrations: $5 \%$ to $25 \%, 26 \%$ to $45 \%, 46 \%$ to $65 \%$ and $68 \%$ to $85 \%$, respectively ${ }^{19}$. In clinical practice, it is difficult to use concentrations above $50 \%$ for the high incidence of side effects, such as nausea, anxiety and vomiting, also documented by other authors 7. A different study has shown a mild decrease in the level of responsiveness, classified as disorientation, with $30 \% \mathrm{~N}_{2} \mathrm{O}$ ${ }^{20}$. Other authors have observed that $20 \%$ and $30 \% \mathrm{~N}_{2} \mathrm{O}$ may impair explicit memory, immediate consciousness and subjective sensations ${ }^{21,22}$.

OAA/S variations with both concentrations (30\% and $50 \%)$ were not clinically important since all patients remained conscious and able to respond to verbal commands.

These observations are in line with other studies ${ }^{23}$ where awaken $\mathrm{N}_{2} \mathrm{O}$ MAC was 0.64 MAC and in this concentration consciousness is not completely abolished. BIS variations are compatible with the weak sedative effect of $30 \%$ and $50 \%$ $\mathrm{N}_{2} \mathrm{O} / \mathrm{SEF} 1$ and SEF2 analysis has shown higher potency density in low frequency ranges, showing that $\mathrm{N}_{2} \mathrm{O}$ had an important slowing-down effect in brain waves frequency. Power energy, another EEG parameter, had a statistical behavior similar to that described by other authors, who have shown an increase in PE as anesthesia goes deeper ${ }^{17}$. Values different than zero in burst suppression variables were not ob- served, showing that in such concentrations, $\mathrm{N}_{2} \mathrm{O}$ is unable to decrease EEG voltage amplitude to below $5 \mu \mathrm{V}^{18}$.

$\mathrm{SBP}, \mathrm{DBP}, \mathrm{HR}$ and $\mathrm{SpO}_{2}$ variations in all moments did not overcome normal pre-established values for the protocol. $\mathrm{N}_{2} \mathrm{O}$ effects on respiratory parameters were in line with the classic description of its effects on spontaneous ventilation with an increase in respiratory rate and decrease in $\mathrm{P}_{\mathrm{ET}} \mathrm{CO}_{2}{ }^{4}$. Our study has differed in minute ventilation, which significantly increased along all moments.

$\mathrm{N}_{2} \mathrm{O}$ at $30 \%$ and $50 \%$ in $\mathrm{O}_{2}$ in patients not premedicated has determined significant BIS, SEF1, SEF2, power energy and $\mathrm{OAA} / \mathrm{S}$ changes. Most significant changes were seen in SEF1 and SEF2, reaching values compatible with deep hypnosis, but with no correspondence with clinical evaluation based on OAA/S or with BIS variation.

Hans et al. ${ }^{24}$, have shown in a recent study, that during surgical procedures, nitrous oxide $(20 \%, 40 \%$ and $60 \%)$ causes a significant dose-dependent BIS and SEF 95\% decrease, with no important change in hemodynamic parameters (BP and $\mathrm{HR}$ ). These authors, however, have associated $\mathrm{N}_{2} \mathrm{O}$ to sufentanil, propofol, sevoflurane and epidural opioid, thus impairing a better validation of results.

As a conclusion, in our conditions, $30 \%$ and $50 \% \mathrm{~N}_{2} \mathrm{O}$ resulted in a weak sedative effect, preserving consciousness. BIS variations were compatible with OAA/S changes, the same not being true for SEF1 and SEF2, which were not valid as quantitative indices of the level of hypnosis but could reflect a possible nitrous oxide analgesic component.

\section{REFERÊNCIAS - REFERENCES}

01. Cahalan MK, Prakash O, Rulf et al - Addition of nitrous oxide to fentanyl anesthesia does not induce myocardial ischemia in patients with ischemic heart disease. Anesthesiology, 1987;67: 925-929.

02. Notini-Gudmarsson AK, Dolk A, Jakobsson J et al - Nitrous oxide: a valuable alternative for pain relief and sedation during routine colonoscopy. Endoscopy, 1996;28:283-287.

03. Wilson S - A survey of the American Academy of Pediatric Dentistry membership; nitrous oxide and sedation. Pediatr Dent, 1996;18:287-293.

04. Cheung AT, Longnecker DE - Pharmacology of Inhalational Anesthetics, em: Crawen L - Principles and Practice of Anesthesiology. USA. Mosby-Year Book. 1998;1123-1157.

05. Tomi K, Mashimo T, Tashiro C et al - Alterations in pain threshold and psychomotor response associated with subanaesthetic concentrations of inhalation anesthetics in humans. Anesthesiology, 1993;70:684-686.

06. Dwyer R, Bennett HL, Eger II El et al - Effects of isoflurane and nitrous oxide in subanesthetic concentrations on memory and responsiveness in volunteers. Anesthesiology, 1992;77: 888-898.

07. Rampil IJ, Kim JS, Lenhardt R et al - Bispectral EEG Index during nitrous oxide administration. Anesthesiology, 1998;89:671-677.

08. Ebert TJ - Differential effects of nitrous oxide on baroreflex control of heart rate and peripheral sympathetic nerve activity in humans Anesthesiology, 1990;72:16-22.

09. Ebert TJ, Kampine JP - Nitrous oxide augments sympathetic outflow: direct evidence from human peroneal nerve recordings. Anesth Analg, 1989;69:444-449. 
10. Hornbein TF Eger II El, Winter PM et al - The minimum alveolar concentration of nitrous oxide in man. Anesth Analg, 1982;61:553-556.

11. Yamamura T, Fukuda M, Takeya H et al - Fast oscillatory EEG activity induced by analgesic concentrations of nitrous oxide in man. Anesth Analg, 1981;60:283-288.

12. Nunes RR, Cavalcante SL, Ibiapina RC et al - Effects of sevoflurane plus fentanyl in hemodynamic, endocrine and electroencephalographic responses to tracheal intubation $\mathrm{Br} J$ Anesthesiol Int Issue, 2001;12:1-6.

13. Kearse LAJ, Rosow C, Zaslavsky A et al - Bispectral analysis of the electroencephalogram predicts conscious processing of information during propofol sedation and hypnosis. Anesthesiology, 1998;88:25-34.

14. Glass PSA, Bloom M, Kearse I et al - Bispectral analysis measures sedation and memory effects of propofol, midazolam, isoflurane and alfentanil in healthy volunteers. Anesthesiology, 1997;86:846-847

15. Nunes RR, Cavalcante SI, Zeferino T - Influência da clonidina na anestesia inalatória com sevoflurano em adultos. Avaliação pelo índice bispectral. Rev Bras Anestesiol, 1999;49:89-93.

16. Chernik DA, Gillings D, Laine $\mathrm{H}$ et al - Validity and reliability of the observer's assessment of alertness/sedation scale: study with intravenous midazolam. J Clin Psychopharmacol, 1990;10:244-251.

17. Schwender D, Daunderer M, Klasing S et al - Power spectral analysis of the electroencephalogram during increasing end-expiratory concentrations of isoflurane, desflurane and sevoflurane. Anaesthesia, 1998;53:335-342.

18. Rampil IJ - A primer for EEG signal processing in anesthesia. Anesthesiology, 1998;89:980-1002.

19. Parbrook GD - Therapeutic use of nitrous oxide: a review. $\mathrm{Br} \mathrm{J}$ Anaesth, 1968;366-372.

20. Galinkin JL, Janiszewski D, Young CJ et al - Subjective, psychomotor, cognitive and analgesic effects of subanesthetic concentrations of sevoflurane and nitrous oxide. Anesthesiology, 1997;87:1082-1088.

21. Henrie JR, Parkhouse J, Bickford RG - Alterations of human consciousness by nitrous oxide as assessed by eletroencephalography and psychological test. Anesthesiology, 1961;22: 247-259.

22. Cook TL, Smith M, Starkweather JA et al - Behavioral effects of trace and subanesthetic halothane and nitrous oxide en man. Anesthesiology, 1978;49:419-424.

23. Dwyer R, Bennett HL, Eger II El et al - Effects of isoflurane and nitrous oxide in subanesthetic concentrations on memory and responsiveness in volunteers. Anesthesiology, 1992;77:888-898.

24. Hans $\mathrm{P}$, Bonhomme $\mathrm{V}$, Benmansour $\mathrm{H}$ et al - Effect of nitrous oxide on the bispectral index and the $95 \%$ spectral edge frequency of the electroencephalogram during surgery. Anaesthesia, 2001;56:999-1002.

\section{RESUMEN}

Cavalcante SL, Nunes RR - Evaluación de los Parámetros Derivados del Electroencefalograma durante Administración de Diferentes Concentraciones de Óxido Nitroso

Justificativa y Objetivos - El análisis espectral del electroencefalograma viene siendo usado como medida de la profundidad anestésica, nivel de hipnosis y sedación de diversos agentes anestésicos. El objetivo de este estudio fue evaluar los efectos del $\mathrm{N}_{2} \mathrm{O}$ sobre los parámetros derivados del electroencefalograma, nivel de sedación por la Escala Analógica de Alerta y Sedación (EAS), variables hemodinámicas y ventilatorias.

Método - Participaron del estudio 30 pacientes adultos, de ambos sexos, estado físico ASA I, con edades entre 20 y 40 años, que se sometieron al siguiente protocolo: respirar espontáneamente y relajar de ojos cerrados durante diez minutos, siendo colectados datos en tres momentos: M1 - antes de la oferta de $\mathrm{N}_{2} \mathrm{O} ; \mathrm{M} 2-\mathrm{N}_{2} \mathrm{O}$ a $30 \%$ en $\mathrm{O}_{2} ; \mathrm{M} 3-\mathrm{N}_{2} \mathrm{O}$ a $50 \%$ en $\mathrm{O}_{2}$. La colecta de los datos de M2 y M3 fue realizada después de quince minutos de la estabilización de las fracciones expiradas de $\mathrm{N}_{2} \mathrm{O}\left(\mathrm{FeN}_{2} \mathrm{O}\right)$ en $30 \%$ y $50 \%$ respectivamente, con el tiempo de 5 minutos entre las fracciones. Los parámetros estudiados fueron los siguientes: electroencefalográficos: BIS, SEF1, SEF2, potencia total (PT) y tasa de supresión (TS); hemodinámicos: frecuencia cardíaca (FC), presión arterial sistólica (PAS), presión arterial diastólica (PAD); ventilatorios: $\mathrm{SpO}_{2}, \mathrm{P}_{E T} \mathrm{CO}_{2}$, ventilación minuto y frecuencia respiratoria; clínicos: los pacientes fueron clasificados numéricamente en orden decreciente (5, 4, 3, 2 y 1) cuanto a su estado de alerta, por la EAS.

Resultados - El $\mathrm{N}_{2} \mathrm{O}$ a $30 \%$ y a $50 \%$ modificó estadísticamente el BIS, SEF1, SEF2, PT y el grado de sedación por la EAS, al largo de los momentos estudiados. No fueron observadas modificaciones clínicamente importantes, pues todos los pacientes se mostraron cooperativos y levemente sedados, a pesar de las variaciones del SEF1 y SEF2 indicaren valores compatibles con práctica de hipnosis profunda. Las variaciones estadísticas en los parámetros hemodinámicos y ventilatorios no fueron clínicamente significativas.

Conclusiones - El $\mathrm{N}_{2} \mathrm{O}$ a $30 \%$ y a $50 \%$, en pacientes sin medicación pré-anestésica, inducen a un leve estado de sedación evaluado por la EAS, habiendo correspondencia con el BIS, el mismo no ocurriendo con SEF1 y SEF2. 